University of Nebraska - Lincoln

DigitalCommons@University of Nebraska - Lincoln

6-19-2009

\title{
Analytic Description of the High-Energy Plateau in Harmonic Generation by Atoms: Can the Harmonic Power Increase with Increasing Laser Wavelengths?
}

\author{
M. V. Frolov \\ Voronezh State University \\ N. L. Manakov \\ Voronezh State University, manakov@phys.vsu.ru \\ T. S. Sarantseva \\ Voronezh State University \\ M. Yu. Emelin \\ Institute of Applied Physics, Russian Academy of Sciences \\ M. Yu. Ryabikin \\ Institute of Applied Physics, Russian Academy of Sciences \\ See next page for additional authors
}

Follow this and additional works at: https://digitalcommons.unl.edu/physicsstarace

Part of the Physics Commons

Frolov, M. V.; Manakov, N. L.; Sarantseva, T. S.; Emelin, M. Yu.; Ryabikin, M. Yu.; and Starace, Anthony F., "Analytic Description of the High-Energy Plateau in Harmonic Generation by Atoms: Can the Harmonic Power Increase with Increasing Laser Wavelengths?" (2009). Anthony F. Starace Publications. 168. https://digitalcommons.unl.edu/physicsstarace/168

This Article is brought to you for free and open access by the Research Papers in Physics and Astronomy at DigitalCommons@University of Nebraska - Lincoln. It has been accepted for inclusion in Anthony F. Starace Publications by an authorized administrator of DigitalCommons@University of Nebraska - Lincoln. 


\section{Authors}

M. V. Frolov, N. L. Manakov, T. S. Sarantseva, M. Yu. Emelin, M. Yu. Ryabikin, and Anthony F. Starace 


\title{
Analytic Description of the High-Energy Plateau in Harmonic Generation by Atoms: Can the Harmonic Power Increase with Increasing Laser Wavelengths?
}

\author{
M. V. Frolov, ${ }^{1}$ N. L. Manakov, ${ }^{1}$ T. S. Sarantseva, ${ }^{1}$ M. Yu. Emelin, ${ }^{2}$ M. Yu. Ryabikin, ${ }^{2}$ and Anthony F. Starace ${ }^{3}$ \\ ${ }^{1}$ Department of Physics, Voronezh State University, Voronezh 394006, Russia \\ ${ }^{2}$ Institute of Applied Physics, Russian Academy of Sciences, Nizhny Novgorod, Russia \\ ${ }^{3}$ Department of Physics and Astronomy, The University of Nebraska, Lincoln, Nebraska 68588-0111, USA
}

(Received 27 February 2009; published 16 June 2009; publisher error corrected 18 June 2009)

A closed-form analytic formula for high-order harmonic generation (HHG) rates for atoms (that generalizes an HHG formula for negative ions [M. V. Frolov et al., J. Phys. B 42, 035601 (2009)]) is used to study laser wavelength scaling of the HHG yield for harmonic energies in the cutoff region of the HHG plateau. We predict increases of the harmonic power for HHG by $\mathrm{Ar}, \mathrm{Kr}$, and $\mathrm{Xe}$ with increasing wavelength $\lambda$ over atom-specific intervals of $\lambda$ in the infrared region, $\lambda \sim(0.8-2.0) \mu \mathrm{m}$.

For over two decades, the generation of harmonics of intense femtosecond laser radiation by atoms and molecules has been one of the most studied processes in intense laser physics. More recently, high-order harmonic generation (HHG) has become fundamentally important for a wide range of diverse applications. In particular, it is a key component of attosecond science, providing a means to produce attosecond XUV pulses [1]. It also underlies tabletop sources of coherent soft x-ray radiation in important energy regions, such as the "water window" (cf., e.g., Ref. [2], which reports the generation of 300 and $450 \mathrm{eV}$ harmonics of the driving laser wavelength, $\lambda=1.6 \mu \mathrm{m}$ ). In this regard, investigation of the scaling of the HHG yield with increasing $\lambda$ is of great interest (cf. Refs. [3-7]). Finally, very promising are recent applications of HHGbased methods for extracting field-free atomic and molecular data, as in tomographic imaging of molecular orbitals $[8,9]$ and in the extraction of atomic photorecombination cross sections (PRCSs) from HHG experimental data [10]. These latter applications are based on an intuitive parametrization of HHG rates (based on the well-known three-step HHG scenario [11,12]) in terms of the PRCS and an "electron wave packet" (EWP) resulting from the ionization and laser-acceleration steps of the active electron within the three-step scenario. As shown in Refs. [13,14], this parametrization is well supported by direct numerical solutions of the time-dependent Schrödinger equation (TDSE) for a single active electron. However, the analytic structure of the EWP remains a "black box."

In this Letter we present a closed form analytic formula for the HHG rate for harmonics at the high-energy end of the HHG plateau. Included is an analytic formula for the EWP, which is largely independent of the atomic target (in agreement with numerical results of Refs. [13,14]). We use our analytic results to analyze the wavelength scaling of the HHG yield in the region of the HHG plateau cutoff. We find that the scaling law for the yield of harmonics near the cutoff in rare gases is different from that predicted in
Ref. [3] (and partly supported experimentally [6]) for harmonics below the plateau cutoff. Moreover, we show that in some cases the HHG efficiency increases with increasing $\lambda$ in the long-wavelength domain.

To deduce our analytic results for the emission of highharmonic photons by an electron bound in an atomic (Coulomb) potential that interacts with a laser electric field $\mathbf{F}(t)=\hat{\mathbf{z}} F \cos \omega t$ (where $F$ and $\omega$ are the field amplitude and frequency), we discuss first our recent analytic result for HHG rates, $\mathcal{R}\left(E_{\Omega}\right)\left(E_{\Omega}=\hbar \Omega=n \hbar \omega\right)$, for the case of an electron bound by a short-range potential in the state $\psi_{\kappa l m}(\mathbf{r})$, with energy $E_{0}=-\hbar^{2} \kappa^{2} /\left(2 m_{e}\right)$ and angular momentum $l$ [15]. This latter result was derived quantum mechanically [in the tunneling limit, $\gamma \ll 1$, where $\gamma=$ $\hbar \omega /\left(e F \kappa^{-1}\right)$ is the Keldysh parameter] based on a general, ab initio formulation for the HHG amplitude [16] that was applied to the case of HHG by an electron in a short-range potential using time-dependent effective range (TDER) theory [17]. To generalize these short-range potential results to the case of a long-range (Coulomb) potential, we represent Eq. (28) for $\mathcal{R}\left(E_{\Omega}\right)$ in Ref. [15] as a product of three factors,

$$
\mathcal{R}\left(E_{\Omega}\right)=\mathcal{I}(\tilde{F}, \omega) \mathcal{W}(E) \sigma^{(r)}(E), \quad E=E_{\Omega}-\left|E_{0}\right|,
$$

and interpret each of them within the three-step scenario.

(i) The dimensionless "ionization factor," $I(\tilde{F}, \omega)$, is

$$
I(\tilde{F}, \omega)=\frac{4 \gamma^{2}}{(2 l+1) \pi \kappa v_{\text {at }}} \Gamma_{\text {st }}(\tilde{F}), \quad v_{\text {at }}=e^{2} / \hbar,
$$

where $\tilde{F}=F\left|\cos \tilde{\tau}_{0}\right|=0.951 F$ is an "effective" static electric field, where $\tilde{\tau}_{0}$ defines the moment of ionization, $t_{i}=\tilde{\tau}_{0} \omega^{-1}=-0.45 T(T=2 \pi / \omega)$, within the classical three-step HHG scenario. $\Gamma_{\text {st }}(\tilde{F})$ is the decay rate for a weakly bound state $\psi_{\kappa l m=0}(\mathbf{r})$ in a static field $\tilde{\mathbf{F}}$ [18]: 


$$
\Gamma_{\mathrm{st}}(\tilde{F})=\frac{\left|E_{0}\right|}{\hbar}(2 l+1) C_{\kappa l}^{2} \frac{\tilde{F}}{2 F_{0}} e^{-2 F_{0} /(3 \tilde{F})}
$$

where $F_{0}=\sqrt{8 m_{e}\left|E_{0}\right|^{3}} /(e \hbar)=\left(\kappa a_{0}\right)^{3} F_{\text {at }}, a_{0}$ is the Bohr radius, $F_{\text {at }}=5.14 \times 10^{9} \mathrm{~V} / \mathrm{cm}$, and $C_{\kappa l}$ is the dimensionless coefficient in the asymptotic form of $\psi_{\kappa l m}(\mathbf{r})$ :

$$
\left.\psi_{\kappa l m}(\mathbf{r})\right|_{\kappa r \gg 1}=C_{\kappa l} \sqrt{\kappa} r^{-1} e^{-\kappa r} Y_{l m}(\hat{\mathbf{r}}) .
$$

(ii) The "propagation factor," $\mathcal{W}(E)$ (measured in $\mathrm{cm}^{-2} \mathrm{~s}^{-1}$ ), describes the propagation of an ionized electron in the laser field $\mathbf{F}(t)$ from the time of its ionization, $t_{i}$, to the time of its recombination, $t_{r}=\tilde{\tau} \omega^{-1}=0.2 T$ :

$$
\mathcal{W}(E)=\frac{p}{m_{e}} \frac{\left(\delta I_{\mathrm{at}} / I\right)^{2 / 3} \mathrm{Ai}^{2}(\xi)}{\left(v_{\mathrm{at}} \Delta t\right)^{3}},
$$

where $p=\sqrt{2 m_{e} E}, \Delta t \equiv t_{r}-t_{i}=4.086 \omega^{-1}=0.65 \mathrm{~T}$, $\delta=1.866, I$ is the laser intensity, and $I_{\text {at }}=3.51 \times$ $10^{16} \mathrm{~W} / \mathrm{cm}^{2}$. The Airy function's argument,

$$
\xi=\left(\delta I_{\text {at }} / I\right)^{1 / 3}\left(E-\mathcal{E}_{\max }\right) / E_{\mathrm{at}}, \quad E_{\mathrm{at}}=e^{2} / a_{0},
$$

involves the difference between the electron energy $E$, corresponding to a given harmonic energy $E_{\Omega}$, and the maximum energy, $\mathcal{E}_{\max }=3.17 u_{p}+0.32\left|E_{0}\right|$ [where $u_{p}=$ $e^{2} F^{2} /\left(4 m_{e} \omega^{2}\right)$ is the ponderomotive energy], gained from the laser field by the ionized electron along the shortest closed trajectory for the return time $\Delta t[15,19]$. [Note that the HHG amplitude in terms of Airy functions (with arguments different from $\xi$ ) and their first derivatives was obtained earlier in Ref. [20] for the bound $s$ state in a zero-range potential model. However, neither the arguments of these Airy functions nor other factors in the HHG amplitude were presented explicitly.]

(iii) The factor $\sigma^{(r)}(E)$ in Eq. (1) is the TDER result for the differential PRCS for an electron with momentum $\mathbf{p}=$ $p \hat{\mathbf{z}}$ recombining to the bound state $\psi_{\kappa l m=0}(\mathbf{r})$ with emission of a harmonic photon (of energy $E_{\Omega}$ ), whose polarization is the same as that of the laser field $\mathbf{F}(t)$ [15]:

$$
\sigma^{(r)}(E)=\alpha^{3} C_{\kappa l}^{2}\left(\frac{3}{4}\right)^{l} \frac{\left(q^{2}-l\right)^{l+1}}{q\left(q^{2}+1\right)} a_{0}^{2}, \quad q=p /(\hbar \kappa),
$$

where $\alpha=e^{2} /(\hbar c)$. Note that the PRCS in (7) is equivalent to that in the Born approximation. This is because in the TDER theory the electron interacts with the binding potential only through the $l$-wave scattering phase $\delta_{l}(E)$, while the $(l \pm 1)$-wave continuum channels (which contribute to the PRCS according to dipole selection rules) remain undistorted by the short-range potential.

The analytic result for $\mathcal{R}\left(E_{\Omega}\right)$ provides HHG rates for the high-energy end of the HHG plateau that are in excellent agreement with exact TDER results for negative ions (cf. Ref. [15]). As each of the three factors in Eq. (1) has a transparent physical meaning within the three-step HHG scenario [which, as is commonly accepted, does not depend on the atomic species], one can expect that an appropriate generalization of Eq. (1) should give an acceptable description of HHG for atoms. This generalization consists in the replacement of two of the three factors in Eq. (1) by their corresponding atomic counterparts:

$$
\mathcal{R}\left(E_{\Omega}\right)=I_{a}(\tilde{F}, \omega) \mathcal{W}(E) \sigma_{a}^{(r)}(E),
$$

where the propagation factor $\mathcal{W}(E)$ is unchanged, because Eq. (5) is essentially independent of the atomic structure, describing free-electron motion in the laser field $\mathbf{F}(t)$. The TDER PRCS (7) should be replaced by the PRCS $\sigma_{a}^{(r)}$ for a specific atom. For instance, for the ground state hydrogen atom, $\sigma_{a}^{(r)}$ is given by [21]

$$
\sigma_{a}^{(r)}(E)=32 \pi \alpha^{3} \frac{e^{-4 q^{-1} \arctan (q)}}{q^{2}\left(q^{2}+1\right)^{2}\left(1-e^{-2 \pi / q}\right)} a_{0}^{2},
$$

where $q=p a_{0} / \hbar$. Finally, the "Coulomb-modified" ionization factor $I_{a}(\tilde{F}, \omega)$ is given by [cf. Eq. (2)]

$$
I_{a}(\tilde{F}, \omega)=\frac{4 \gamma^{2}}{(2 l+1) \pi \kappa_{a} v_{\mathrm{at}}} \Gamma_{\mathrm{st}}^{(a)}(\tilde{F}),
$$

where $\Gamma_{\text {st }}^{(a)}$ is the tunneling rate for a bound state $\psi_{\kappa_{a} l m=0}(\mathbf{r})$ of an active atomic electron with energy $E_{a}=$ $-\left(\hbar \kappa_{a}\right)^{2} /\left(2 m_{e}\right)$ in a static electric field $\tilde{\mathbf{F}}$ [18]:

$$
\Gamma_{\mathrm{st}}^{(a)}(\tilde{F})=\frac{\left|E_{a}\right|}{\hbar}(2 l+1) C_{\kappa_{a} l}^{2} l\left(\frac{2 F_{a}}{\tilde{F}}\right)^{2 \nu-1} e^{-2 F_{a} /(3 \tilde{F})},
$$

where $\nu=Z /\left(\kappa_{a} a_{0}\right), Z$ is the charge of the atomic core ( $Z=1$ for neutral atoms), $F_{a}=\left(\kappa_{a} a_{0}\right)^{3} F_{\text {at }}$, and $C_{\kappa_{a} l}$ is given by the asymptotic form of $\psi_{\kappa_{a} l m}(\mathbf{r})$ [cf. Eq. (4)]:

$$
\left.\psi_{\kappa_{a} l m}(\mathbf{r})\right|_{\kappa_{a} r \gg 1}=C_{\kappa_{a} l} \sqrt{\kappa_{a}} r^{-1}\left(\kappa_{a} r\right)^{\nu} e^{-\kappa_{a} r} Y_{l m}(\hat{\mathbf{r}}) .
$$

We stress that only the PRCS in Eq. (8) is sensitive to the energy-dependent atomic dynamics, while the EWP,

$$
W(E)=I_{a}(\tilde{F}, \omega) \mathcal{W}(E),
$$

contains only two "static" atomic parameters, $\left|E_{a}\right|$ and $C_{\kappa_{a} l}$. Equations (5) and (10) show clearly that $W(E)$ represents the flux of recombining electrons with velocity $v=$ $p / m_{e}$. Thus Eqs. (1) and (8) justify analytically the $a d$ hoc parametrization of the HHG yield in Refs. $[13,14]$.

To test the accuracy of Eq. (8), we compare our analytic results with results of numerical solutions of the TDSE for a hydrogen atom subjected to a trapezoidal laser pulse with a 16-cycle flat top of intensity $I=2 \times 10^{14} \mathrm{~W} / \mathrm{cm}^{2}$ and two-cycle ramps for turn-on or -off. The TDSE was solved by using the FFT split-operator spectral method [22] and the imaginary potential method in order to absorb reflection of waves from the grid boundary [23]. The accuracy of the numerical solution of the TDSE was monitored by increasing the grid size and by decreasing the grid cell. [The final results were obtained for a grid cell of $\left(0.4 a_{0}\right)^{3}$ over a box of $100 a_{0}$ in both the $x$ and $y$ axes and up to $z \gtrsim$ $6 e F /\left(m_{e} \omega^{2}\right)$ in the direction of laser polarization. A fixed mesh width in $t$ was used: $\Delta t=0.48$ a.u.] As an additional 
test, we have checked that the harmonic power, $P=$ $E_{\Omega} \mathcal{R}\left(E_{\Omega}\right)$, integrated over the interval $20 \leq E_{\Omega} \leq$ $50 \mathrm{eV}$ decreases with increasing $\lambda$ over the interval $0.8-1.6 \mu \mathrm{m}$ as $\lambda^{-x}$ with $x \approx 5.2$. This result is close to the result $x \approx 4.8$ of Ref. [4] for an eight-cycle flat-top sine pulse with a half-cycle turn-on and turn-off and $I=1.6 \times$ $10^{14} \mathrm{~W} / \mathrm{cm}^{2}$.

Figure 1 shows good agreement of the TDSE and analytic results at the high-energy ends of the HHG spectra. The accuracy of the analytic result (8) is better for larger $\lambda$ [since Eq. (1) was derived in [15] in the low-frequency (long-wavelength) limit]. Since the PRCS (9) varies smoothly with energy, the oscillatory pattern along the high-energy plateau in Fig. 1 maps interference oscillations of the EWP, $W(E)$, as a function of $E_{\Omega}$ (cf. Ref. [15] for details). Both our analytic results and the TDSE results in Fig. 1 show that the harmonic power at the cutoff scales differently from the widely accepted law $P(\lambda) \sim \lambda^{-3}$ $[19,24]$. According to Eqs. (2) and (5), the EWP, $W(E)$, decreases for large $\lambda$ as $\lambda^{-4}$, whereas the PRCS at the HHG cutoff energy $\left(E_{\Omega}=E_{c} \approx\left|E_{a}\right|+3.17 u_{p} \sim \lambda^{2}\right)$ gives an extra dependence on $\lambda$. In the limit $\lambda \rightarrow \infty$, the argument of the PRCS in Eq. (8) goes to infinity, so that the Born approximation may be used for $\sigma_{a}^{(r)}(E)$. Thus, $\sigma_{a}^{(r)} \sim$ $E^{-5 / 2} \sim \lambda^{-5}$ for a Coulomb potential, while $\sigma^{(r)} \sim \lambda^{2 l-1}$ for a short-range potential [cf. Eq. (7)]. Hence, $P(\lambda)$ scales as $\lambda^{-7}$ for a Coulomb field and $\lambda^{2 l-3}$ for a short-range potential. Thus, the $\lambda^{-3}$-scaling is realized only for an $s$ state in a short-range potential (to which, in fact, the analyses in Refs. [19,24] correspond). In our analysis above we assume $\lambda \rightarrow \infty$ (or $\gamma \rightarrow 0$ ), while for $\lambda$ in the interval from 1.0 to $1.6 \mu \mathrm{m}$, the Keldysh parameter varies from $\gamma=0.60$ to 0.38 for $I=2 \times 10^{14} \mathrm{~W} / \mathrm{cm}^{2}$. Thus, the scaling law $\lambda^{-6.1}$ for $\lambda=1.0 \mu \mathrm{m}$ and $1.6 \mu \mathrm{m}$, observed in Fig. 1, is slightly less steep than the asymptotic one, $\lambda^{-7}$.

The situation is different for nonhydrogenic atoms. For these atoms, the photoionization cross section (PICS), which is related to the PRCS by the equation of detailed

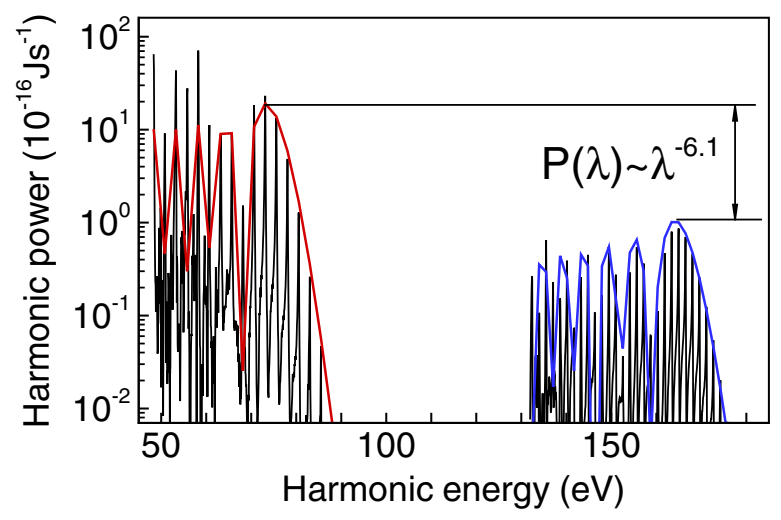

FIG. 1 (color online). HHG spectra for the $\mathrm{H}$ atom for $I=$ $2 \times 10^{14} \mathrm{~W} / \mathrm{cm}^{2}$ and two laser-field wavelengths, $\lambda=1.0 \mu \mathrm{m}$ (left) and $\lambda=1.6 \mu \mathrm{m}$ (right). Thin lines: numerical TDSE results; thick lines: analytic results obtained using Eq. (8). balance [21], exhibits irregularities caused by Cooper minima, potential barrier and electron correlation effects, etc. [25]. Owing to these irregularities, a universal scaling law for $P(\lambda)$ is not possible. Moreover, they can affect the interference structures in the HHG spectra shown in Fig. 1.

As the oscillations in $P(\lambda)$ [considered as a function of harmonic energy $E_{\Omega}$ (cf. Fig. 1)] are smoothed when integrated over $E_{\Omega}$, the energy-integrated HHG power (i.e., that of a group of near-cutoff harmonics) should elucidate more definitively the atomic dynamic effects caused by irregularities of $\sigma_{a}^{(r)}(E)$. When $P(\lambda)$ was integrated over $E_{\Omega}$ in an energy interval $\Delta E$ below the cutoff (from 20 to $50 \mathrm{eV}$ ), it was found numerically to satisfy the following scaling law [3]: $P_{\Delta E}(\lambda) \sim \lambda^{-(5-6)}$. We consider here the harmonic power integrated over a fixed energy interval $\Delta E$ centered at the cutoff energy $E_{c}\left(\approx\left|E_{a}\right|+\right.$ $\left.3.17 u_{p}\right)$ :

$$
\begin{aligned}
P_{\Delta E}(\lambda) & =\hbar \omega \sum_{n_{-}}^{n_{+}} n \mathcal{R}\left(E_{\Omega}\right) \\
& \approx \frac{1}{2 \hbar \omega} \int_{E_{c}-\Delta E / 2}^{E_{c}+\Delta E / 2} E_{\Omega} \mathcal{R}\left(E_{\Omega}\right) d E_{\Omega},
\end{aligned}
$$

where $n_{ \pm}=\left[\left(E_{c} \pm \Delta E / 2\right) /(\hbar \omega)\right]$, and $[x]$ is the integer part of $x$. Using Eq. (8), the integral in Eq. (14) may be approximated for the case of small $\Delta E[\Delta E<$ $2 E_{\mathrm{at}}\left(\delta I_{\mathrm{at}} / I\right)^{-1 / 3}$ ] by taking into account that the argument of the Airy function $\operatorname{Ai}(\xi)$ in Eq. (5) for this case varies over the interval $-1<\xi<+1$ for $E_{\Omega}$ in the interval $E_{c} \pm$ $\Delta E / 2$ [cf. Eq. (6)]. Since $\operatorname{Ai}(\xi)$ is smooth in the interval $|\xi|<1$, it may be replaced by a constant, e.g., $\mathrm{Ai}(0)$. As a result, Eq. (14) (after changing the integration variable to $\left.E=E_{\Omega}-\left|E_{a}\right|\right)$ gives

$$
P_{\Delta E}(\lambda) \sim \frac{1}{\lambda^{4}} \int_{3.17 u_{p}-\Delta E / 2}^{3.17 u_{p}+\Delta E / 2} E^{3 / 2} \sigma_{a}^{(r)}(E) d E .
$$

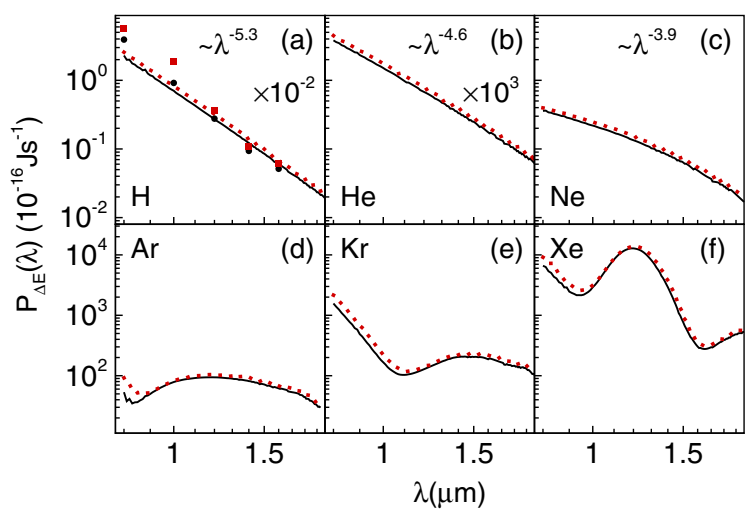

FIG. 2 (color online). Harmonic power, $P_{\Delta E}(\lambda)$ [cf. Eq. (14)], vs $\lambda$ for $\mathrm{H}$ and the rare gases with $I=2 \times 10^{14} \mathrm{~W} / \mathrm{cm}^{2}$ and two energy intervals $\Delta E$ : 20 (solid lines) and $30 \mathrm{eV}$ (dotted lines). Circles and squares in panel (a) mark the TDSE results for $\Delta E=$ 20 and $30 \mathrm{eV}$, respectively. 
This expression shows clearly that the $\lambda$-scaling of $P_{\Delta E}(\lambda)$ for harmonic energies near the cutoff (for a given $\lambda$ ) is sensitive to the energy dependence of $\sigma_{a}^{(r)}(E)$ in the interval $3.17 u_{p} \pm \Delta E / 2$.

For both short-range and Coulomb potentials, the PICSs decrease smoothly with increasing $E$, giving a smooth dependence of $P_{\Delta E}(\lambda)$ on $\lambda$. Indeed, in the longwavelength limit, the Born result for $\sigma_{a}^{(r)}(E)$ in Eq. (9) may be used in Eq. (15). That gives $P_{\Delta E}(\lambda) \sim \lambda^{-6}$ for the case of a Coulomb potential, while the PRCS (7) gives $P_{\Delta E}(\lambda) \sim \lambda^{2 l-2}$ for a short-range potential. For rare gases, the result is different. In Fig. 2 we present $P_{\Delta E}(\lambda)$ for $\mathrm{H}$ and the rare gases obtained by using Eq. (8) and summing over $n$ in Eq. (14) numerically. We present also the exact results for $\mathrm{H}$ [using TDSE results for $\mathcal{R}\left(E_{\Omega}\right)$ ], which show the accuracy of using the analytic result (8) for $\mathcal{R}\left(E_{\Omega}\right)$ in Eq. (14). The PRCS data for recombination to the outer $s$ shell of He and outer $p$-shells of other rare gases were deduced from the PICSs data for these shells found in Refs. [26] (He), [27] (Ne), [28] (Ar), [29] (Kr), and [30] (Xe). For $\mathrm{H}, \mathrm{He}$, and $\mathrm{Ne}$, the PRCSs are smooth in the considered interval of energy $E$, leading to a smooth decrease of $P_{\Delta E}(\lambda)$ with increasing $\lambda$, approximately as $\lambda^{-5.3}, \lambda^{-4.6}$, and $\lambda^{-3.9}$, respectively. For $\mathrm{Ar}$ and $\mathrm{Kr}$, the PICSs have Cooper minima, which lead to minima in $P_{\Delta E}(\lambda)$ near $\lambda=0.9$ and $1.1 \mu \mathrm{m}$. Finally, the result for Xe shows a broad maximum. This maximum is a "multielectron replica" of the known "giant" resonance in the PICS from the inner $4 d$ shell in Xe that appears in the PICS from the outer $5 p$ shell due to interchannel couplings [30]. Therefore, for the heavy rare gases, the dependence of $P_{\Delta E}(\lambda)$ on $\lambda$ is irregular and can increase with increasing $\lambda$ in the long-wavelength region.

To conclude, Eqs. (1) and (8) for HHG rates provide closed-form, analytic quantum expressions of the famous semiclassical three-step scenario for the HHG process, having the same level of transparency and simplicity as the Keldysh result for tunnel ionization. The three factors in Eq. (8) describe, respectively, the "atomic" processes of ionization and recombination $\left[I_{a}(\tilde{F}, \omega)\right.$ and $\left.\sigma_{a}^{(r)}(E)\right]$ and the laser-driven propagation of the ionized electron $[\mathcal{W}(E)]$. The EWP, $W(E)$, in Eq. (13) is not sensitive to the energy-dependent atomic dynamics; it describes all oscillatory structures in the HHG spectrum, which originate from interference of two (short and long) classical electron trajectories (cf. Ref. [15]). Using Eq. (8), the $\lambda$-scaling law for the integrated power of near-cutoff harmonics is shown to be sensitive to the energy dependence of the atom's PRCS. This dependence provides a means to increase the frequency of harmonics without significant loss, or even a possible gain, in the HHG efficiency by increasing the laser wavelength into an optimum interval for a particular atomic target.

This work was supported in part by RFBR Grant No. 0702-00574, by NSF Grant PHY-0601196, by Russian President's Grant No. 1931.2008.2, and by the
"Dynasty” Foundation (T. S. S. and M. Yu. E).

[1] P. Agostini and L. F. DiMauro, Rep. Prog. Phys. 67, 813 (2004); F. Krausz and M. Ivanov, Rev. Mod. Phys. 81, 163 (2009).

[2] E. J. Takahashi, T. Kanai, K. L. Ishikawa, Y. Nabekawa, and K. Midorikawa, Phys. Rev. Lett. 101, 253901 (2008).

[3] J. Tate et al., Phys. Rev. Lett. 98, 013901 (2007).

[4] K. Schiessl, K. L. Ishikawa, E. Persson, and J. Burgdörfer, Phys. Rev. Lett. 99, 253903 (2007); K. L. Ishikawa, K. Schiessl, E. Persson, and J. Burgdörfer, Phys. Rev. A 79, 033411 (2009).

[5] M. V. Frolov, N. L. Manakov, and A. F. Starace, Phys. Rev. Lett. 100, 173001 (2008).

[6] P. Colosimo et al., Nature Phys. 4, 386 (2008).

[7] D. B. Milošević et al., J. Mod. Opt. 55, 2653 (2008).

[8] J. Itatani et al., Nature (London) 432, 867 (2004).

[9] J. Levesque, D. Zeidler, J. P. Marangos, P. B. Corkum, and D. M. Villeneuve, Phys. Rev. Lett. 98, 183903 (2007).

[10] S. Minemoto et al., Phys. Rev. A 78, 061402(R) (2008).

[11] K. J. Schafer, B. Yang, L. F. DiMauro, and K. C. Kulander, Phys. Rev. Lett. 70, 1599 (1993).

[12] P. B. Corkum, Phys. Rev. Lett. 71, 1994 (1993).

[13] T. Morishita, A. T. Le, Z. Chen, and C. D. Lin, Phys. Rev. Lett. 100, 013903 (2008).

[14] A. T. Le, T. Morishita, and C.D. Lin, Phys. Rev. A 78, 023814 (2008).

[15] M. V. Frolov, N. L. Manakov, T. S. Sarantseva, and A. F. Starace, J. Phys. B 42, 035601 (2009).

[16] M. V. Frolov, A. V. Flegel, N.L. Manakov, and A.F. Starace, Phys. Rev. A 75, 063407 (2007).

[17] M. V. Frolov, A. V. Flegel, N.L. Manakov, and A.F. Starace, Phys. Rev. A 75, 063408 (2007).

[18] B. M. Smirnov and M. I. Chibisov, Zh. Eksp. Teor. Fiz. 49, 841 (1965) [Sov. Phys. JETP 22, 585 (1966)].

[19] M. Lewenstein, Ph. Balcou, M. Yu. Ivanov, A. L'Huillier, and P. B. Corkum, Phys. Rev. A 49, 2117 (1994).

[20] D. B. Milošević and W. Becker, Phys. Rev. A 66, 063417 (2002).

[21] I. I. Sobel'man, Introduction to the Theory of Atomic Spectra (Elsevier, Oxford, 1972).

[22] J. A. Fleck, Jr., J. R. Morris, and M. D. Feit, Appl. Phys. 10, 129 (1976); M. D. Feit, J. A. Fleck, Jr., and A. Steiger, J. Comput. Phys. 47, 412 (1982).

[23] J. L. Krause, K. J. Schafer, and K. C. Kulander, Phys. Rev. A 45, 4998 (1992).

[24] W. Becker, S. Long, and J. K. McIver, Phys. Rev. A 50, 1540 (1994).

[25] A. F. Starace, in Handbuch der Physik, edited by S. Flügge and W. Mehlhorn (Springer-Verlag, Berlin, 1982), Vol. XXXI, p. 1; M. Ya. Amusia, Atomic Photoeffect (Plenum, NY, 1990).

[26] K. L. Bell, A. E. Kingston, and I. R. Taylor, J. Phys. B 6, 1228 (1973).

[27] T. N. Chang and T. Olsen, Phys. Rev. A 23, 2394 (1981).

[28] J. Tulkki, Phys. Rev. A 48, 2048 (1993).

[29] J. Tulkki et al., Phys. Rev. A 45, 4640 (1992).

[30] M. Kutzner, V. Radojević, and H. P. Kelly, Phys. Rev. A 40, 5052 (1989). 